\title{
Fidelity of Output Images in One-Way Image Transmission through a Distorting Medium Using Phase Conjugation
}

\author{
Huitian WANG, Jiasen ZHANG, Shin YOSHIKADO, and Tadashi ARUGA \\ Communications Research Laboratory, Ministry of Posts and Telecommunications \\ 4-2-1 Nukui-Kitamachi, Koganei, Tokyo 184-8795
}

(Received September 8, 1997)

\begin{abstract}
We investigated and explain herein the dependence of the fidelity of the reconstructed output images on the position of the distorting medium for one-way image transmission through a thin distorting medium by using the wave-front reversal advantage of four-wave mixing phase conjugation in a photorefractive crystal.
\end{abstract}

Key Words: One-way image transmission, Fidelity, Phase conjugation, Four-wave mixing, Photorefractive effect.

\section{Introduction}

Optical phase conjugation has an impressive fascination because it can make severely distorted optical beam or image to be reconstructed to original, unaberrated state. This distinctive property suggests a great lot of potential applications in many areas, such as adaptive optics, ${ }^{1)}$ optical resonators, ${ }^{2)}$ interferometer, ${ }^{3)}$ spatial mode cleanup of laser beam, ${ }^{4)}$ optical information processing, ${ }^{5)}$ measurement of surface granularity, ${ }^{6}$ etc. Its another important application, which is image transmission, is always provoked some researcher's interest because the spatial image or the beam are degraded due to propagating in optical fibers, atmosphere, or inhomogenous distorting medium. In past years, the image transmission through a single fiber has been investigated by using phase conjugation in several experiments, ${ }^{7-9)}$ but they have a common disadvantage that the image reconstructed is situated at the same side of the fiber as the input image, in other words, the image cannot be transferred from one place to another, although the image was well reproduced. Yariv ${ }^{10)}$ proposed a method by using a pair of optical fibers and phase conjugator for one-way image transmission (not round-trip), which was recently demonstrated experimentally. ${ }^{11)}$ However there still exists obvious difficulty or disadvantages that the two fibers should be exactly the same in size, shape, and optical properties, as well as the displacements of the incident position and the angle between the two fibers.

We are of interest for one-way image transmission through a thin distorting medium in space. Yariv and co-workers presented a proposal and an analysis for one-way coherent image transmission through a distorting medium using four-wave mixing ${ }^{12)}$ and demonstrated experimentally real-time phase conjugate window for one-way optical field image transmission through a distorting medium. ${ }^{13)}$ In this paper, we investigate and explain the dependence of the fidelity of the reconstructed output images on the position of the distorting medium for one-way image transmission through a thin distorting medium.

\section{Experiment}

The experimental scheme is illustrated in Fig.1. Light source used in this experiment is a frequency-doubled Nd:YAG pulsed laser operating at a wavelength of $532 \mathrm{~nm}$ with a pulse width of $3.0 \mathrm{~ns}$ and a repetition rate of $80 \mathrm{~Hz}$. The output of laser is collimated and expanded, and then the central portion is cut out by a diaphragm to obtain a beam as uniform as possible which is diverged by beam splitters to produce three beams 1,2 and 4 . The nonlinear material used is a cerium-doped barium titanate $\left(\mathrm{BaTiO}_{3}: \mathrm{Ce}\right)$ crystal which had dimensions of $10 \times 7.3 \times 1.5$ $\mathrm{mm}^{3}$ with $c$-axis along the edge of $10 \mathrm{~mm}$. Beam 4 and two counterpropagating beams 1 and 2 interact by four-wave mixing configuration in the crystal to generate beam 3 counterpropagating to beam 4 . Beams 1,2 and 4 have a intensity ratio of $I_{1}$ : $I_{2}: I_{4}=4: 1: 2\left(I_{2}=100 \mathrm{~mW} / \mathrm{cm}^{2}\right)$. The two $4 \times$ telescope systems composed of lenses $\mathrm{L}_{\mathrm{j} 1}$ and $\mathrm{L}_{\mathrm{j} 2}$ (focal lengths: $f_{\mathrm{j} 1}=80$ $\mathrm{cm}, f_{\mathrm{j} 2}=20 \mathrm{~cm}, j=2$ and 4 ) are respectively interposed in beampaths 2 and 4 . There have a distance of $f_{\mathrm{j} 2}$ between the crystal and $\mathrm{L}_{\mathrm{j} 2}$. An input image is situated at the front focal plane of $\mathrm{L}_{21}$ in order to obtain the true replica of the input image on the back focal plane of $\mathrm{L}_{22}$ (i.e., on the crystal) and avoid the loss of the spatial information. A thin phase distorting medium (DM), which is formed by smearing thin transparent glue on a microscope slide, is placed at a plane $\mathrm{D}$ with a coordinate system $\left(x_{\mathrm{D}}, y_{\mathrm{D}}\right)$ attached in beam-path 4 . We use the variable $d$ to represent a distance between the plane $\mathrm{D}$ and the front focal plane $\mathrm{F}$ of $\mathrm{L}_{41}$,

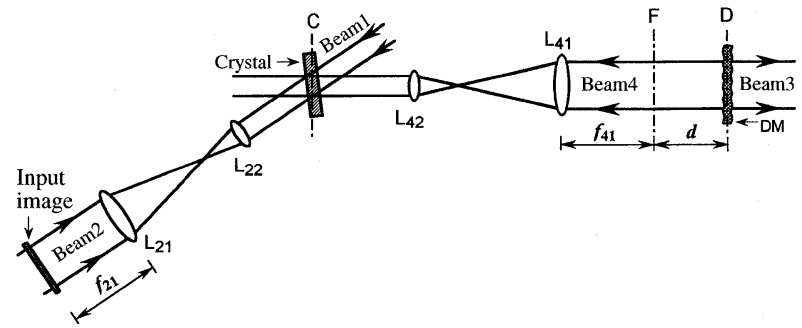

Fig.1 Experimental scheme for one-way image transmission through a thin phase distorting medium. 
and assume $d$ to be positive if the plane $\mathrm{D}$ is in the right of the plane F.

\section{Results and Explanation}

Firstly we examine the deformed patterns of the image-bearing beam and an uniform beam after passing through DM. Figure 2 (a) shows the original input image. Figure 2 (b) and 2 (d) are respectively the destroyed patterns of the image-bearing beam and the uniform beam for 3-cm distance behind DM, and Figs. 2 (c) and 2 (e) are respectively the corresponding destroyed patterns for $10-\mathrm{cm}$ distance behind DM. It can be seen that DM makes the input image and the uniform beam to be deformed thoroughly.

Secondly we carry out the experiment of the one-way image transmission using the configuration in Fig.1. The experimental results are shown in Fig.3. Figs.3 ow $1 \sim$ ow 4 are the oneway output images corresponding to four different positions of $\mathrm{DM}, d=20 \mathrm{~cm}, 0,-2 \mathrm{~cm}$, and $-20 \mathrm{~cm}$, respectively. It is obvious that the output images are well distinguishable although there have some incompleteness or lack of fidelity. We can also see
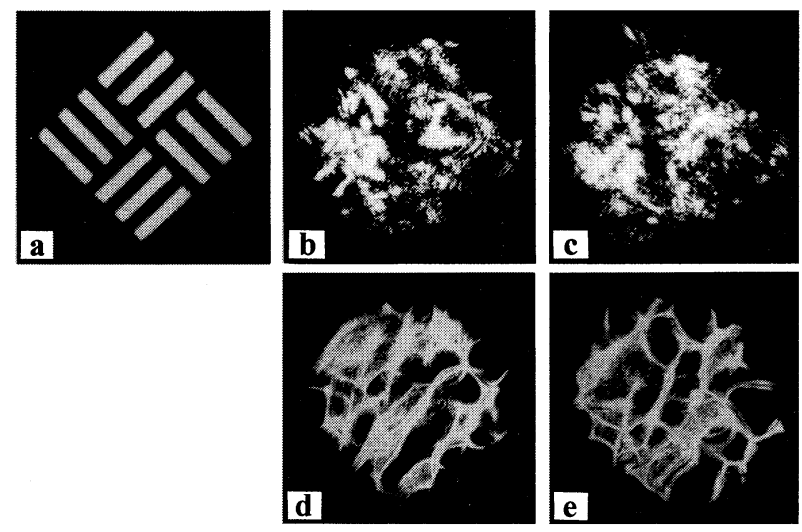

Fig.2 Input image and patterns deformed after passing through the distorting medium. a: input image pattern; $b$ and $d$ : the deformed patterns of input image and uniform beam at places of $3 \mathrm{~cm}$ behind the distorting medium, respectively; $\mathrm{c}$ and e: corresponding patterns at places of $10 \mathrm{~cm}$. that Fig. 3 ow2, which corresponds to that DM is situated at the plane $\mathrm{F}(d=0)$, has the highest fidelity among Figs.3 ow1 ow4 and is clearly discernible and nearly without any distorting medium. From the experimental results it is shown that the input image can be transmitted from the left side of DM to the right, in other words, one-way image transmission through a distorting medium has been achieved. Fig. 3 ow 5 is the output image when there are no any distorting media in all beam-paths. The fidelity of the output image without DM (Fig.3 ow5) is higher than that with DM (Figs.3 ow1 ow4). When DM deviate from the plane $\mathrm{F}$, the one-way output image becomes worse and worse. This effect will be interpreted in the following.

For the convenience of understanding, we assume that two back focal planes of $\mathrm{L}_{22}$ and $\mathrm{L}_{42}$ are parallel (namely, beams 2 and 4 are co-linear) although there is a cross-angle. That is to say, two back focal planes of $\mathrm{L}_{22}$ and $\mathrm{L}_{42}$ are coincided at the same plane, which is referred as a plane C. A coordinate system $\left(x_{\mathrm{C}}, y_{\mathrm{C}}\right)$ is attached to the plane $\mathrm{C}$, with the coordinate axes parallel with those in the plane D. Actually, DM may be regarded as a diffraction grating, but it is a grotesquely unconventional phase grating without any periodicity. If using $\varphi\left(x_{\mathrm{D}}, y_{\mathrm{D}}\right)$ to express the phase distribution of DM, the complex amplitude transmittance of DM may be

$$
t_{\mathrm{D}}\left(x_{\mathrm{D}}, y_{\mathrm{D}}\right)=\exp \left[j \varphi\left(x_{\mathrm{D}}, y_{\mathrm{D}}\right)\right]
$$

An uniform beam with amplitude $A$ illuminates DM, then the disturbance of the field on the plane $D$ is

$$
U_{\mathrm{D}}\left(x_{\mathrm{D}}, y_{\mathrm{D}}\right)=A t_{\mathrm{D}}\left(x_{\mathrm{D}}, y_{\mathrm{D}}\right) \equiv A \exp \left[j \varphi\left(x_{\mathrm{D}}, y_{\mathrm{D}}\right)\right]
$$

In accordance with the Huygens-Fresenl principle, the disturbance at a point $\left(x_{\mathrm{C}}, y_{\mathrm{C}}\right)$ on the plane $\mathrm{C}$ may be regarded as a convolution of $U_{\mathrm{D}}\left(x_{\mathrm{D}}, y_{\mathrm{D}}\right)$ with an impulse response function $h\left(x_{\mathrm{C}}, y_{\mathrm{C}} ; x_{\mathrm{D}}, y_{\mathrm{D}}\right)$; that is,

$$
U_{\mathrm{C}}\left(x_{\mathrm{C}}, y_{\mathrm{C}}\right)=\iint_{\mathrm{D}} h\left(x_{\mathrm{C}}, y_{\mathrm{C}} ; x_{\mathrm{D}}, y_{\mathrm{D}}\right) U_{\mathrm{D}}\left(x_{\mathrm{D}}, y_{\mathrm{D}}\right) \mathrm{d} x_{\mathrm{D}} \mathrm{d} y_{\mathrm{D}},
$$

and

$$
\begin{gathered}
h\left(x_{\mathrm{C}}, y_{\mathrm{C}} ; x_{\mathrm{D}}, y_{\mathrm{D}}\right)=Q \iint_{-\infty}^{+\infty} \exp \left[j \pi \lambda^{-1}\left(f_{42}^{-1}-M f_{41} f_{42}^{-2}\right)\left(x^{2}+y^{2}\right)\right] \\
\exp \left\{-j 2 \pi\left[\left(M x_{\mathrm{D}}+x_{\mathrm{C}}\right) x+\left(M y_{\mathrm{D}}+y_{\mathrm{C}}\right) y\right]\right\} \mathrm{d} x \mathrm{~d} y,
\end{gathered}
$$
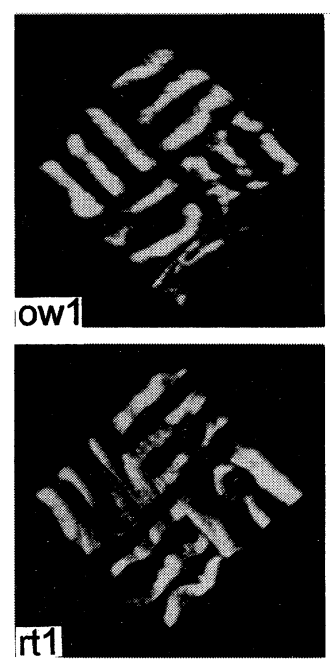
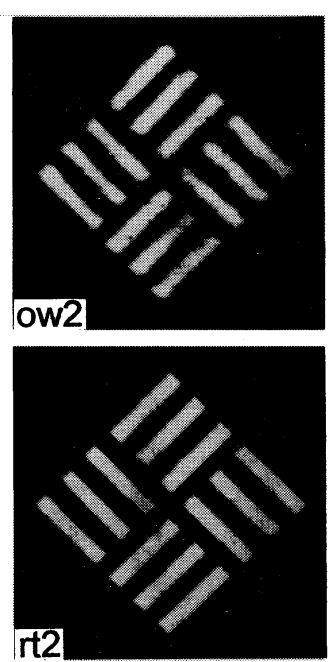
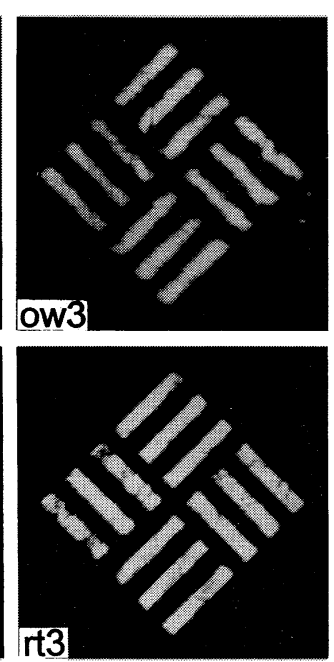
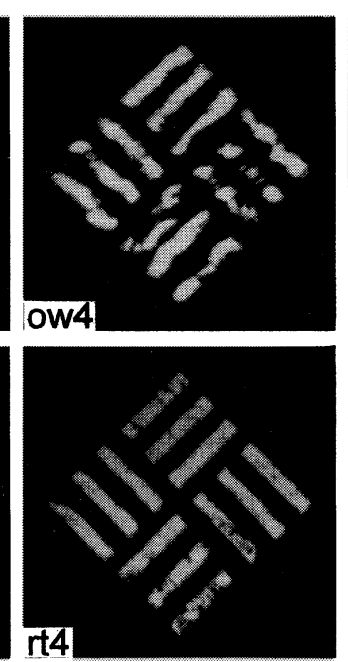
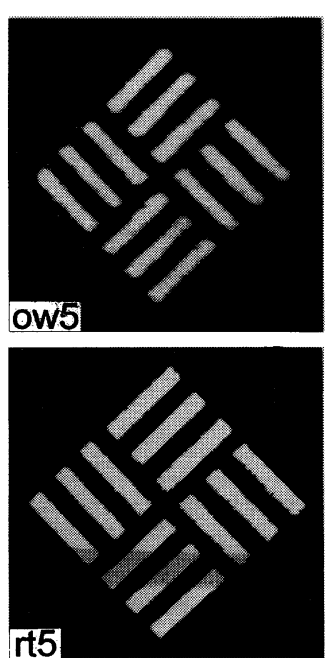

Fig.3 Experimental results. ow1 ow5: one-way output images, rt1 rt5: round-trip output images. ow1, rt1: $d=20 \mathrm{~cm}$; ow2, rt2: $d=0$; ow3, rt3: $d=-2 \mathrm{~cm}$, ow $4, \mathrm{rt} 4: d=-20 \mathrm{~cm}$; ow5, rt5: without distorting medium. 


$$
M=f_{41} f_{42}\left[f_{41}^{2}-\mathrm{d} f_{42}\right]^{-1}
$$

where $Q$ is constant and $\lambda$ is the wavelength. With the above equations $(1) \sim(5)$, it can be seen that, in general, the disturbance at point $\left(x_{\mathrm{C}}, y_{\mathrm{C}}\right)$ on the plane $\mathrm{C}$ is determined by superposition of the disturbances of all points on the plane D. However, if the plane $\mathrm{C}$ is the image-plane of the plane $\mathrm{D}$ with respect to the telescope system composed of $\mathrm{L}_{41}$ and $\mathrm{L}_{42}$, namely when the plane $\mathrm{D}$ is situated at the front focal plane $\mathrm{F}$ of $\mathrm{L}_{41}(d=0)$, at this time, the impulse response function $h$ reduces to a simple $\delta$ function

$$
\left.h\left(x_{\mathrm{C}}, y_{\mathrm{C}} ; x_{\mathrm{D}}, y_{\mathrm{D}}\right)\right|_{\mathrm{d}=0}=Q \delta\left(M_{0} x_{\mathrm{D}}+x_{\mathrm{C}}, M_{0} y_{\mathrm{D}}+y_{\mathrm{C}}\right),
$$

where

$$
\left.M_{0} \equiv M\right|_{\mathrm{d}=0}=f_{42} / f_{41}
$$

As a result, with (3) and (6) the disturbance at point $\left(x_{\mathrm{C}}, y_{\mathrm{C}}\right)$ on the plane $\mathrm{C}$ is

$$
\left.U_{\mathrm{C}}\left(x_{\mathrm{C}}, y_{\mathrm{C}}\right)\right|_{\mathrm{d}=0}=\frac{Q}{M_{0}} U_{\mathrm{D}}\left(-\frac{x_{\mathrm{C}}}{M_{0}},-\frac{y_{\mathrm{C}}}{M_{0}}\right) .
$$

From the equation (8), we can find that when DW is placed at the plane $\mathrm{F}$, the disturbance at point $\left(x_{\mathrm{C}}, y_{\mathrm{C}}\right)$ on the plane $\mathrm{C}$ is solely determined by its corresponding object point $\left(x_{\mathrm{D}}, y_{\mathrm{D}}\right)$ on the plane $\mathrm{D}$ and is independent of any other points. From the viewpoint of information processing, if the plane $\mathrm{C}$ is the image-plane of the plane $\mathrm{D}$, then point $\left(x_{\mathrm{C}}, y_{\mathrm{C}}\right)$ on the plane $\mathrm{C}$ carries only all information of its corresponding object point $\left(x_{\mathrm{D}}\right.$, $y_{\mathrm{D}}$ ) on the plane $\mathrm{D}$, but not any information of other points; otherwise it will bear partial information of all points on the plane D. As mentioned before, for the case of Fig. 3 ow2, which is corresponding to the case that the plane $\mathrm{C}$ is the image-plane of the plane $\mathrm{D}$, point $\left(x_{\mathrm{C}}, y_{\mathrm{C}}\right)$ on the plane $\mathrm{C}$ carries all information of its object point $\left(x_{\mathrm{D}}, y_{\mathrm{D}}\right)$ on the plane $\mathrm{D}$, but not any information of any other points. If point $\left(x_{\mathrm{C}}, y_{\mathrm{C}}\right)$ on the plane $\mathrm{C}$ overlaps with the bright region of image-bearing beam 2 , all information carried by point $\left(x_{\mathrm{C}}, y_{\mathrm{C}}\right)$ can be conjugated and returned to its corresponding object point $\left(x_{\mathrm{D}}, y_{\mathrm{D}}\right)$, thus the distorting medium will be completely corrected or canceled. On the other hand, if point $\left(x_{\mathrm{C}}, y_{\mathrm{C}}\right)$ coincides with dark regions, then no phase conjugate signal is produced. Therefore, an undistorted replica of the input image can be reconstructed after passing through DM. Now we see the other cases corresponding to Figs. 3 ow 1, ow 3 and ow4, namely DM deviates from the plane $\mathrm{F}$. In one aspect, point $\left(x_{\mathrm{C}}, y_{\mathrm{C}}\right)$ on the plane $\mathrm{C}$ contains partial information of every point on the plane $\mathrm{D}$. If point $\left(x_{\mathrm{C}}, y_{\mathrm{C}}\right)$ overlaps with the dark region of beam 2, no signal can be generated, thus this part of information of all points on the plane $\mathrm{D}$ related to point $\left(x_{\mathrm{C}}, y_{\mathrm{C}}\right)$ will vanish. As a result, the distortion cannot be thoroughly restored although the generated signal beam 3 retraces the telescope system and DM. In another aspect, there appear the nonuniform intensity diffraction pattern on the plane $\mathrm{C}$, especially the intensity is very weak or even zero in some regions (see Figs.2 (d) and 2 (e). When these regions overlap with the bright region of beam 2 , then those information of the input image will be lost because the phase conjugate signal cannot be generated. Therefore, the output image is inferior and cannot be improved by increasing the apertures of beams when $d \neq 0$. This is the reason why the fidelity of the output images becomes lower and lower when DM gradually departures from the plane $\mathrm{F}$.

In addition, we also observed that the horizontal dimension of one-way output images were slightly different from its vertical dimension, which actually originated from that there exists a cross-angle between beams 2 and 4 . For the photographs in this paper this effect has been corrected by using a cylindrical lens.

In order to compare the one-way image transmission with the round-trip case, we remove the input image from the front focal plane of $L_{21}$ in beam-path 2 to the plane $F$ in beam-path 4 to observed the round-trip output images corresponding to the four different positions of DM mentioned above. The results are shown in Figs. 3 rt1 rt4. Fig. 3 rt5 is the round-trip output image without DM. It is clear that the fidelities of the round-trip output images is better than that of the corresponding one-way output images. Figs. $3 \mathrm{rt} 2$ and $\mathrm{rt} 5$ have very high fidelity and almost no difference comparing with the input image. The fidelity of Fig. $3 \mathrm{rt} 1$ corresponding to $d=20 \mathrm{~cm}$ is evidently lower than others and cannot be improved by increasing the apertures of beams 1 and 2. The reason is that to bear an image on a deformed beam will lose a part of information of the input image. We found also that the fidelities of Figs. $3 \mathrm{rt} 3$ and $\mathrm{rt} 4$ were somewhat lower than that of Fig. $3 \mathrm{rt} 2$. The reason is that the deviation of the position of DM from the plane $F$ cause the difference of the diameters of beam 1 and beam 2 in the crystal, as a result, a part of the information of the input image will be intercepted. This problem can be solved by increasing the apertures of beams 1 and 2 .

\section{Conclusion}

We have demonstrated the one-way image transmission through a thin distorting medium in free-space by using fourwave mixing phase conjugation in a photorefractive crystal. Simultaneously, the dependence of the fidelity of the reconstructed output images on the position of the distorting medium was investigated and explained. The experimental results and analysis indicated that high fidelity one-way reconstructed image can be obtained provided the distorting medium is not thick.

\section{References}

1) V. Wang and C. R. Giuliano: Opt. Lett. 2 (1978) 4.

2) J. F. Lam and W. P. Brown: Opt. Lett. 5 (1980) 61.

3) J. Feinberg: Opt. Lett. 8 (1983) 569.

4) T. Y. Chang: Opt. Lett. 15 (1990) 1342.

5) S. Sternklar, S. Weiss, and B. Fischer: Opt. Eng. 26 (1987) 423.

6) J. Khoury, M. Cronin-Golomb, A. M. Biernacki, and C. L. Woods: Appl. Opt. 33 (1994) 7655.

7) G. J. Dunning and R. C. Lind: Opt. Lett. 7 (1982) 558.

8) B. Fischer and S. Sternklar: Appl. Phys. Lett. 46 (1985) 113.

9) P. H. Beckwith, I. McMichael, and P. Yeh: Opt. Lett. 12 (1987) 510.

10) A. Yariv: Appl. Phys. Lett. 28 (1976) 88.

11) T. Ogasawara, M. Ohno, and K. Karaki: Opt. Lett. 20 (1995) 2435.

12) A. Yariv and T. L. Koch: Opt. Lett. 7 (1982) 113.

13) B. Fischer, M. Cronin-Golomb, J. O. White, and A. Yariv: Appl. Phys. Lett. 41 (1982) 141. 